\title{
Design of an immersive simulator for assisted power wheelchair driving
}

\author{
Louise Devigne $^{1}$, Marie Babel ${ }^{2}$, Florian Nouviale ${ }^{3}$, \\ Vishnu K. Narayanan ${ }^{2}$, François Pasteau ${ }^{4}$ and Philippe Gallien ${ }^{5}$
}

\begin{abstract}
Driving a power wheelchair is a difficult and complex visual-cognitive task. As a result, some people with visual and/or cognitive disabilities cannot access the benefits of a power wheelchair because their impairments prevent them from driving safely. In order to improve their access to mobility, we have previously designed a semi-autonomous assistive wheelchair system which progressively corrects the trajectory as the user manually drives the wheelchair and smoothly avoids obstacles. Developing and testing such systems for wheelchair driving assistance requires a significant amount of material resources and clinician time. With Virtual Reality technology, prototypes can be developed and tested in a risk-free and highly flexible Virtual Environment before equipping and testing a physical prototype. Additionally, users can "virtually" test and train more easily during the development process. In this paper, we introduce a power wheelchair driving simulator allowing the user to navigate with a standard wheelchair in an immersive 3D Virtual Environment. The simulation framework is designed to be flexible so that we can use different control inputs. In order to validate the framework, we first performed tests on the simulator with able-bodied participants during which the user's Quality of Experience (QoE) was assessed through a set of questionnaires. Results show that the simulator is a promising tool for future works as it generates a good sense of presence and requires rather low cognitive effort from users.
\end{abstract}

\section{INTRODUCTION}

Driving a power wheelchair safely requires good environmental perception that is derived from the visual and cognitive abilities of an individual [1]. As a consequence, people who have visual and/or cognitive impairments have an increased risk for accidents and experience difficulties in performing driving tasks. Therefore, they may be prevented from driving a power wheelchair for safety reasons, thus greatly reducing their mobility as well as their opportunity to socialize [2].

To address this issue, smart assistive systems have to be developed in order to facilitate users to navigate effectively without the help from a caregiver.

Testing and adapting such systems requires human and material resources especially for the field tests environment which needs to be mounted and unmounted. To lessen these

\footnotetext{
*This work was not supported by any organization

${ }^{1}$ Louise Devigne is with the Rehabilitation Center Pôle Saint Hélier, the Lagadic Team at IRISA/Inria Rennes and INSA Rennes, France

${ }^{2}$ Marie Babel and Vishnu K. Narayanan are with the Lagadic Team at IRISA/Inria Rennes and INSA Rennes, France marie.babeleirisa.fr

${ }^{3}$ Florian Nouviale is with INSA Rennes, France

${ }^{4}$ François Pasteau is with the Ergovie Company, Rennes, France

${ }^{5}$ Philippe Gallien is with the Rehabilitation Center Pôle Saint Hélier, Rennes, France
}

limitations, Virtual Reality (VR) can reveal itself a powerful tool as it offers a safe and modifiable environment. Indeed, Virtual Reality is being an increasingly used method in rehabilitation as it can have a positive impact on user's motivation [3][4].

Virtual Reality technologies were initially used to provide assistance to people with disabilities [5][6]. The aim of these VR systems was to help people develop new skills such as communication skills [7], to improve their ability to achieve tasks of the daily life such as making groceries at the supermarket [8] or to train for specific tasks such as driving a wheelchair [9].

Such a simulator must provide a realistic environment, perception and interactions in order to reproduce real life situations: the idea is to ease transfer of skills and behaviors acquired during the wheelchair driving sessions.

Besides, some studies have been conducted on the relevance of a simulator for driving. A long-term study on car driving has shown positive transfer of driving skills to real life situations [10]. As for wheelchair driving, a study comparing the wheelchair driving performances of two groups in a real and in a simulated environment [11] showed similar performances for power wheelchair driving in the simulator or in real life. Moreover, [12] highlighted that wheelchair driving performances in the Virtual Environment (VE) can be similar to driving abilities in a real environment. They also emphasized the fact that virtual wheelchair driving training may provide greater practice to users while requiring less clinician time. Indeed, using a Virtual Environment allows to automate data collection thus reducing the need of supervision from medical staff to perform tests as it provides a safe environment without any risk of collision or physical injury for the user. Another benefit of using a simulator for wheelchair driving is that it is extremely flexible as we are not limited in space and as we can modify the existing environment or create a new one very quickly.

Several research teams have developed driving simulators and VR applications with power wheelchair navigation scenarios. They were particularly used to assess wheelchair driving performances and to perform wheelchair driving training [13][14][15][16].

Most of the wheelchair driving simulators are displayed on a computer screen or with a Head Mounted Device (HMD). The Virtual Environment thus generally includes the representation of a self-avatar as it increases the sense of immersion and improves size and distance judgments [17].

Here, we propose to design a driving simulator for power 
wheelchair assisted navigation which can be used with a real power wheelchair placed in a an immersive VR room: as a consequence, the user does not need a self-avatar to judge distances as the VE around the wheelchair is life-size scaled. To this aim, we propose to place a standard wheelchair on a large scale VR platform. In addition to this, we integrated a shared-control algorithm for power wheelchair driving which we designed and presented in [18]. This solution has been tested and validated by regular wheelchair users through clinical trials in the Rehabilitation Center Pôle Saint Hélier. The main objective of this study is to validate the use of the simulator in terms of user quality of experience. To this aim, we conducted an experiment with able-bodied participants who performed wheelchair navigation tasks on the simulator with or without driving assistance.

The paper is organized as follows. Section II outlines the implementation work that has been realized to develop the simulator. Section III presents the experiment that has been conducted with able-bodied participants on the simulator and Section IV presents the results.

\section{System ARCHITECTURE}

\section{A. Simulator}

We initially designed a Virtual Environment in which we can navigate using a real power wheelchair. The development of this framework was achieved in collaboration with the Hybrid team from the Irisa/Inria computer science laboratory. We used the VR platform Immersia (Fig. 1) owned by our laboratory and located in Rennes. This platform is composed of 4 screens on which the 3D images are projected a life-size scaled 3D environment in which we can navigate with a real power wheelchair. With this setup, we do not need to manage user embodiment and issues of avatar control required with Head Mounted Displays.

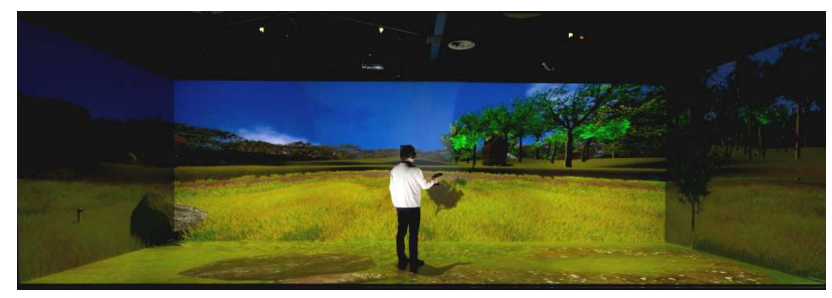

Fig. 1: Immersia is one of the largest VR research platforms in the world. It is composed of 4 large screens on which images are projected for a total volume of approximately $90 \mathrm{~m}^{3}$. The $3 \mathrm{D}$ rendering is achieved by using active stereoscopic method associated with shutter glasses. 16 infrared cameras track the moving objects on the platform.

The idea is to design a 3D VE in which we are able to take control of a virtual wheelchair (Fig. 3). The dimensions and the behavior of the virtual wheelchair have to be rigorously identical to those of the real wheelchair we put on the platform so that we can provide realistic behavior. We use the same wheelchair as in [18], a Luca power wheelchair from the You-Q company (Fig. 2). In this framework, the user is sitting on this wheelchair and controls a standard

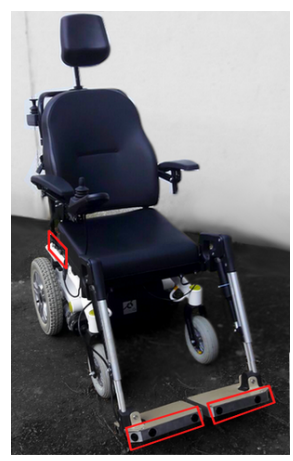

Fig. 2: Real Wheelchair. Red frames indicate ultrasonic sensor positions.

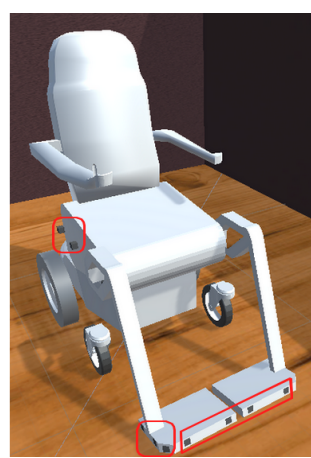

Fig. $\quad 3: \quad$ Virtual

Wheelchair. Red frames indicate virtual sensor positions.

joystick coming from Penny \& Giles to operate the virtual wheelchair.

The user is sitting on a real wheelchair placed on the VR platform (Fig. 4) at the same position as the virtual wheelchair in the VR platform's frame. The user's head position is tracked in order to move the virtual scene viewpoint accordingly to user movements.

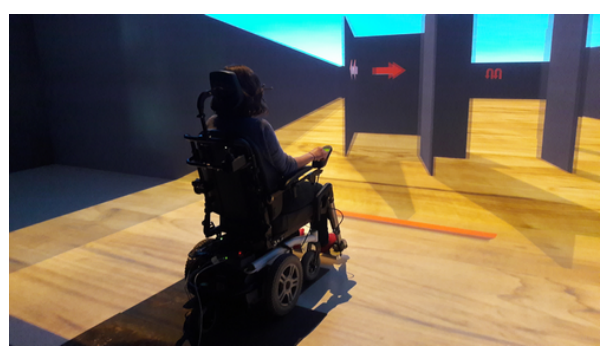

Fig. 4: A participant on the wheelchair placed on the VR platform

\section{B. Implementation work}

The simulator development consisted of creating a VE in which we operate a virtual robotized wheelchair (equipped with virtual sensors). This requires the use of Unity 3D and MiddleVR to be easily interfaced with the Immersia platform. Unity $3 \mathrm{D}$ is a widely used cross-platform game creation system developed by Unity Technologies. It includes a game engine and an integrated development environment. MiddleVR is a generic immersive VR plugin compatible with Unity. The framework is designed to be flexible and is based on ROS middleware. 3 ROS nodes have been developed:

- Unity3D ROS node: VE display, data collection from the VE and application of the commands to the virtual wheelchair, here torques applied for each motor wheel.

- Algorithm ROS node: computation of the commands to be sent to the virtual wheelchair

- Controller ROS node: collection of wheelchair controller data, here the standard wheelchair joystick commands. 
These nodes form a generic framework which could be used with any type of input controller (regular wheelchair joystick, chin control device, game controller...). The principal functionality of this framework is to control a virtual wheelchair (Unity3D node) using a command (Algorithm node) computed from the input controller information (Controller node). Added to this, we can use virtual sensor information (red dashed line) to compute a control law (Algorithm node) either modifying the command sent to the wheelchair to provide obstacle avoidance or sending information to a biofeedback interface (black dashed line). This generic solution provides flexibility and allows to easily switch from the virtual world to the real world by replacing the Unity3D node with a node managing embedded sensor measurement and control on the wheelchair prototype.

A schematic representing the framework architecture is given in Fig. 5.

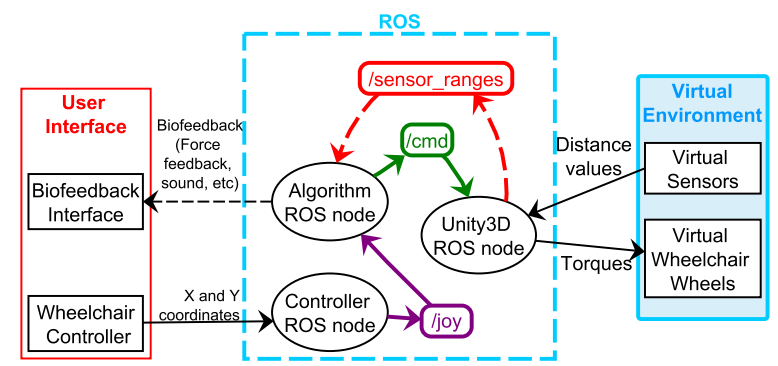

Fig. 5: Input commands are collected using the Controller node and used by the Algorithm node to compute the commands to the virtual wheelchair. The Unity3D node handles the display of the VE as well as the commands to the virtual wheelchair by applying torques to its wheels.

\section{Virtual driving assistance}

We integrated our semi-autonomous driving assistance algorithm presented in [19] in the simulator. As this it is based on distance information, we simulated distance sensors attached to the virtual wheelchair. As for the real wheelchair, the distance sensor we model are ultrasonic car sensors which detect distances within a cone shaped area with a total angle of $60^{\circ}$ (Fig. 6). Each sensor in the VE is then modeled as a virtual object emitting 3 rays (Fig. 7).

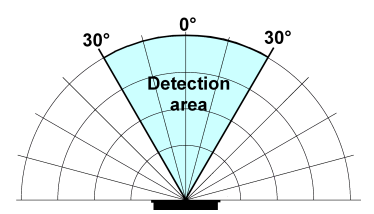

Fig. 6: Ultrasonic sensor cone shaped detection.

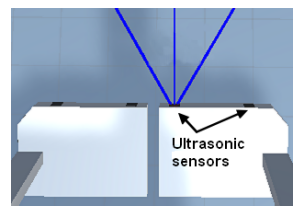

Fig. 7: Virtual sensor in Unity3D. The blue lines represent the rays sent by a sensor
Distance from the virtual sensor to the virtual obstacle is measured using the raycasting technique in Unity which consists of sending one ray from an object in a given direction. Once this ray collides with an obstacle, it is then possible to retrieve related information such as the distance between the 2 objects and the normal of the surface the ray hit.

We could throw more rays for each sensor to measure distance more accurately but the greater the number of rays, the greater the computational cost. Instead, we compute the distance measured by each virtual sensor ray to obtain the distances from the virtual wheelchair to the obstacles. This in particular allows us to handle the case of corner avoidance (Fig. 8). The calculation of the distance is as follows.

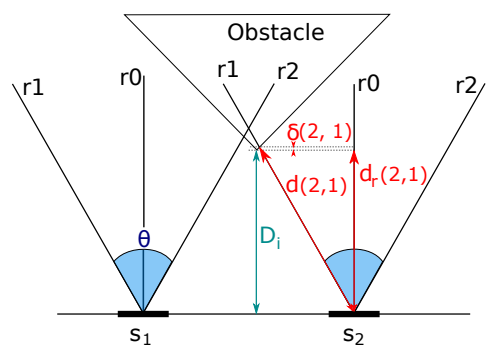

Fig. 8: Particular case of corner detection.

With two sensors $s_{1}$ and $s_{2}, j=0,1,2$ and $i=1,2$, let - $\theta$ be the angle of the cone-shaped detection area,

- $d(i, j)$ be the distance returned by the $j^{t h}$ ray thrown by the virtual sensor $s_{i}$

- $r_{0}, r_{1}$ and $r_{2}$ be the 3 rays sent by each sensor

- $\delta(i, j)$ be the error between the distance measured by the ray $r_{j}$ sent by the virtual sensor $s_{i}$ and the actual distance from the wheelchair to the obstacle.

The distance $d_{r}$ to a corner shaped obstacle positioned is defined as follows :

$\left\{\begin{array}{l}d_{r}(i, j)=d(i, j) \text { if } \mathrm{j}=0, \\ d_{r}(i, j)=d(i, j) \cos \left(\frac{\theta}{2}\right)-\delta(2,1) \simeq d(i, j) \cos \left(\frac{\theta}{2}\right), \text { else. }\end{array}\right.$

Finally, the distance $D_{i}$ measured by the $i^{\text {th }}$ virtual sensor is equal to

$$
D_{i}=\min _{j=0,1,2} d_{r}(i, j) .
$$

This framework enables us to test different sensor configurations more easily and more rapidly than on a real robotized wheelchair prototype. In particular, this allowed us to address more effectively the shortcomings of the system in terms of sensor positioning which we reported in previous work [19]. We empirically determined an optimal configuration composed of 22 sensors.

\section{EXPERIMENT}

\section{A. Experimental protocol}

The purpose of this wheelchair driving simulator is to help us to develop, test and compare wheelchair driving assistance solutions in a VE. In this pilot study, we performed an experiment with able-bodied participants in order to validate 
our framework and assess user quality of experience and satisfaction. The experiment consisted in navigating in the VE while sitting on a real power wheelchair placed on the immersive platform. The VE was composed of two obstacle courses:

- Obstacle course A (Fig. 9): This obstacle course is taken from [20]. It is composed of maneuvers such as right/left turns, discrete maneuverability obstacles, straight line path and a turnaround.

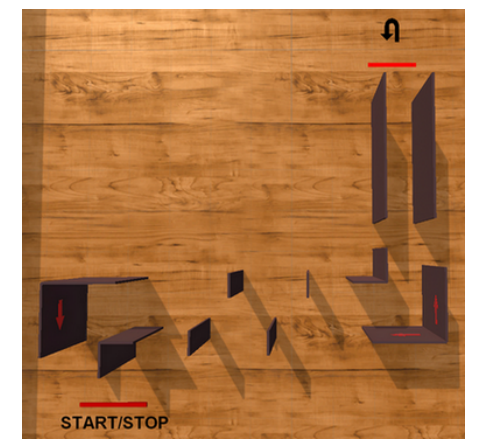

Fig. 9: Obstacle course taken from [20] in the VE.

- Obstacle course B (Fig. 10): The second obstacle course was designed for the experiment. The user starts at the beginning of a corridor of decreasing width, turns right and follows a $1 \mathrm{~m}$ wide corridor with a $0.9 \mathrm{~m}$ wide open door on his right. At the end of the corridor the user passes through a narrower passage and traverses a space with obstacles.

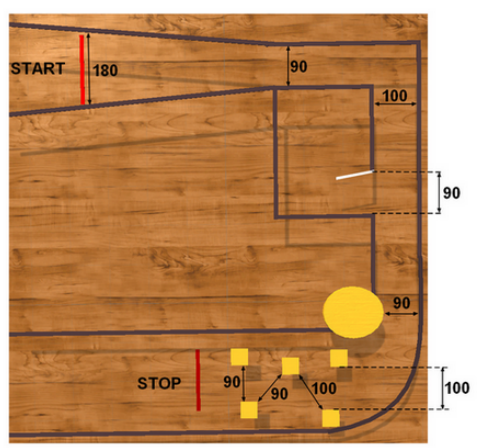

Fig. 10: Obstacle course we designed for the experiment.

Participants were first asked to perform a set of individual driving tasks derived from the widely used standardized Wheelchair Skill Test ([21]) such as rolling forwards, turning $90^{\circ}$ while moving forward, turning in place. This preliminary training session was done in order to ensure that participants were able to manipulate the wheelchair in the VE before accomplishing other tasks in the VE.

After this short training, participants were asked to drive the wheelchair on the two obstacle courses with and without driving assistance in single-blind so that the participants were not noticed if the driving assistance was activated or not. We randomized the trials by switching the order of the driving assistance state of activation. Moreover, we alternated the order of passage for the 2 obstacle courses to avoid a possible learning effect.

The overall test takes about 20 minutes per participant.

\section{B. Data collection}

1) Simulator data: We use ROS to automatically record data during the trials. Here we record controller commands, virtual wheelchair position and speed, collisions, data from virtual sensor and time completion.

2) Questionnaire: After completing the obstacle courses on the simulator, participants are asked to complete a set of questionnaires. The first one is the I-Group Presence Questionnaire (IPQ [22]). This test consists of 14 items divided into 4 categories namely Spatial Presence (SP), Experienced Realism (REAL), Involvement (INV), and General Impression $(G)$ evaluated using a 7-point Likert scale. We also assess the workload imposed by the simulator using the widely used NASA Task Load Index questionnaire (TLX [23]).

\section{RESUlts}

9 able-bodied participants aged from 11 to 38 years old have volunteered to participate to this study. Most of the participants were novice with power wheelchair driving, only one of them rated his experience with power wheelchair driving as 5 out of 6 on a 7-point Likert-type scale.

\section{A. Presence}

Presence was measured using the IPQ Questionnaire [22]. Means and standard deviation for all categories are displayed in Fig. 11. Scores were on average above 3 for Involvement (INV) and above 4 for General Impression (G) and Spatial Presence (SP). Experienced Realism score (REAL) was on average below 3 .

\section{B. Perceived Workload}

Perceived workload was assessed using the NASA-TLX Questionnaire [23]. NASA-TLX scores are displayed on Fig. 12. The results show scores on average below 50 and with a mean of 27.2+/-18.2 (Mean +/- Standard Deviation) over the 9 participants.

\section{Simulator sickness}

6 participants reported having felt sick during and/or after the experiment and 4 of them also suffered from headache. The intensity of these effects was moderate and did not prevent the users from completing the experiment.

\section{Driving assistance}

7 participants over 9 noticed the activation of the driving assistance. Fig. 13 shows the number of collisions that occurred with no assistance activated during the experiments. Participants collided a total number of 25 times. With assistance activated, only 1 participant hit an obstacle.

The completion time values are summarized in Fig. 14 and show that trials with assistance last more time than trials without assitance. 


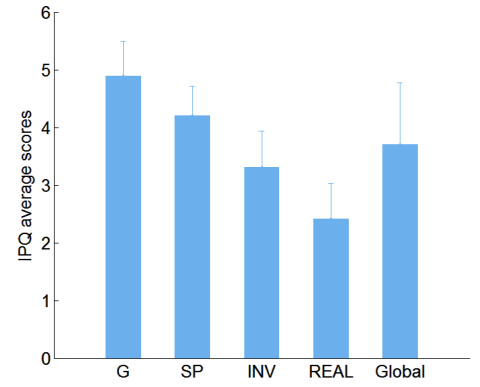

Fig. 11: Average IPQ score for each category. Global represents the global score. Error bars indicate standard deviation.
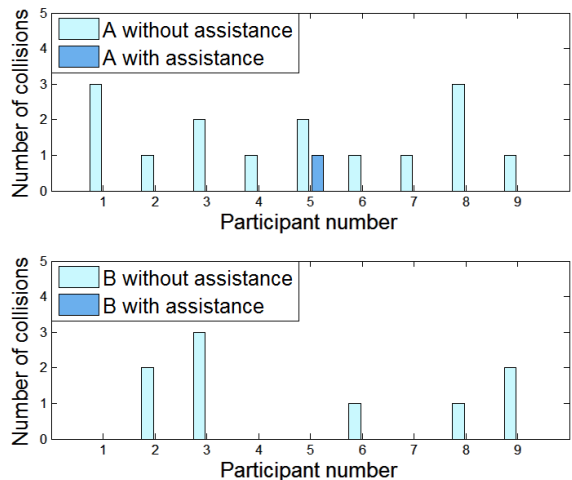

Fig. 13: Number of collisions for each participant on both obstacle courses with and without driving assistance activated.

\section{DISCUSSION}

The purpose of this study was to assess our simulator for assisted power wheelchair driving in terms of user satisfaction. To this aim, we asked 9 able-bodied participants to perform an experiment consisting in driving in the Virtual Environment with a real power wheelchair. This step is essential before we consider tests with people with disability.

Our simulator seemed to be accepted by the participants as it generated a good sense of presence. IPQ scores showed that they felt present in the simulated environment. Indeed, Global score was on average above 3 and General Impression as well as Spatial Presence scores were quite high (on average above 4). Also, the results of the NASA TLX questionnaire suggest that the use of the simulator does not require too much cognitive load. As for the driving assistance, 7 over 9 participants noticed its activation. It is to be noted that most of them were aware of the nature of our research work probably leading to a bias in their perception of driving assistance activation. One of the two other participants who did not notice the activation of the assistance is used to drive a power wheelchair: this tends to show that the behavior of the virtual wheelchair in the simulator is not altered by the application of the driving assistance algorithm.

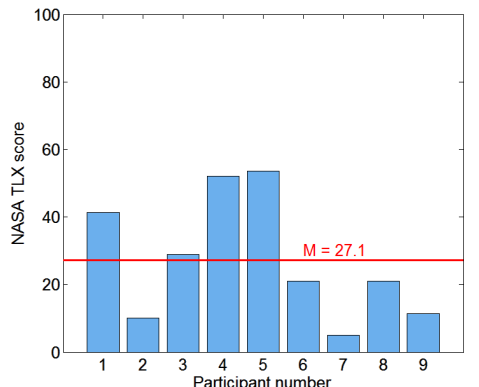

Fig. 12: NASA-TLX scores M represents the mean value over the 9 participants.
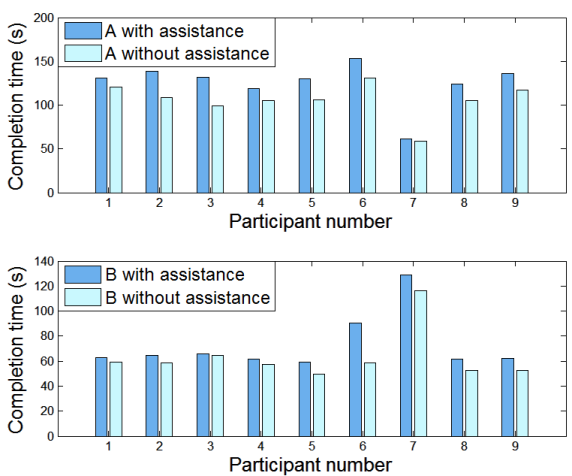

Fig. 14: Completion time for each participant on both obstacle courses with and without driving assistance activated.

Most of the participants reported having felt moderately sick during the navigation tasks. This issue could be partly due to the fact that driving in the VE generates quick movements for which the refresh rate of the virtual reality platform (60 images per second) could be insufficient. For another part, this is due to the fact that the wheelchair is not moving while moving images are displayed on the screens. This probably leads to an alteration of the perceived selfmotion. To address this issue some improvements of the simulator can be made. First, as it is indicated in [24], adding auditory cues can increase the perceived self-motion. In future work we will then address this problem by adding sounds to our simulator, especially wheelchair noises. We could also increase navigation realism and perceived selfmotion by adding tactile sensory cues using a mechanical force feedback platform [24].

Results of the experiments show that participants collided a total of 25 times with no driving assistance whereas only one participant collided once with an obstacle while the assistance was activated. Collisions happened more often on the obstacle course A, particularly on the part with maneuverability obstacles. Here, performing navigational tasks on obstacle courses allowed us to validate the use of virtual sensors to perform driving assistance. The collision recorded while the assistance was activated happened on a 
difficult maneuver where obstacles are discrete and quite thin. Therefore, this collision was most probably due to the fact that the rays sent by virtual sensors did not detect the thin obstacle. One solution to this could be to improve virtual sensor measurement by using cone-shaped virtual objects instead of raycasting method.

\section{CONCLUSION}

In this paper we presented a generic simulator for power wheelchair driving and its first test on the Virtual Reality platform Immersia in Rennes. Such a simulator is directly compliant with a monitor screen display, a Virtual Reality room or a HMD. We choose to implement this framework on a 3D VR platform thus providing a VR simulator in which the user navigates with a standard wheelchair in a life-size scale virtual 3D environment. The simulator was validated through tests performed with able-bodied participants on the Virtual Reality platform in Rennes.

With a powerful tool such as Virtual Reality we aim at designing more ecological situations with situations typically encountered by wheelchair users containing large spaces but also more difficult tasks such as navigation in a narrow corridor or with obstacles on the way. In future work, we will improve the simulator with new Virtual Environments and introduction of more sensory cues to increase the sense of immersion. Also we will use this simulator to go further in our research on power wheelchair driving assistance. Indeed, this simulator has the advantage of providing quick and easy setting as well as requiring significantly less time and material resources. We plan to use this simulator to ease the research process on curb following and biofeedback solutions. We also plan to perform other experiments with people with disability.

\section{ACKNOWLEDGEMENT}

This project has received funding from the European Interreg project ADAPT. The authors would like to thank Ronan Gaugne, Bruno Arnaldi and Valérie Gouranton from the Hybrid team. Also we would like to thank Manutea Huang, Aline Baudry, Valentin Bureau and Nicolas Le Borgne from INSA Rennes who were of great help during the development process and experiments. Finally the authors would like to thank all the people who participated to this study.

\section{REFERENCES}

[1] S. Massengale, D. Folden, P. McConnell, L. Stratton, and V. Whitehead, "Effect of visual perception, visual function, cognition, and personality on power wheelchair use in adults," Assistive Technology, vol. 17, no. 2, pp. 108-121, 2005.

[2] R. C. Simpson, "Smart wheelchairs: A literature review." Journal of rehabilitation research and development, vol. 42, no. 4, pp. 423-436, 2004.

[3] C. Bryanton, J. Bosse, M. Brien, J. Mclean, A. McCormick, and H. Sveistrup, "Feasibility, motivation, and selective motor control: virtual reality compared to conventional home exercise in children with cerebral palsy," Cyberpsychology \& behavior, vol. 9, no. 2, pp. 123-128, 2006.

[4] M. C. Howard, "A meta-analysis and systematic literature review of virtual reality rehabilitation programs," Computers in Human Behavior, 2017.
[5] D. Strickland, L. M. Marcus, G. B. Mesibov, and K. Hogan, "Brief report: Two case studies using virtual reality as a learning tool for autistic children," Journal of Autism and Developmental Disorders, vol. 26, no. 6, pp. 651-659, 1996.

[6] J. McComas, P. Pivik, and M. Laflamme, "Current uses of virtual reality for children with disabilities," Studies in health technology and informatics, pp. 161-169, 1998.

[7] D. Strickland, "Virtual reality for the treatment of autism," Studies in health technology and informatics, pp. 81-86, 1997.

[8] P. Standen and J. Cromby, "Can students with developmental disability use virtual reality to learn skills which will transfer to the real world," in Proceedings of the 1995 CSUN Virtual Reality Conference, 1995.

[9] D. P. Inman and K. Loge, "Teaching motorized wheelchair operation in virtual reality," in Proceedings of the 1995 CSUN Virtual Reality Conference, 1995.

[10] P. Hirsch and F. Bellavance, "Pilot project to validate the transfer of training of driving skills learned on a high fidelity driving simulator to on-road driving," 2016.

[11] P. S. Archambault, S. Tremblay, S. Cachecho, F. Routhier, and P. Boissy, "Driving performance in a power wheelchair simulator," Disability and Rehabilitation: Assistive Technology, vol. 7, no. 3, pp. 226-233, 2012

[12] R. A. Cooper, D. M. Spaeth, D. K. Jones, M. L. Boninger, S. G. Fitzgerald, and S. Guo, "Comparison of virtual and real electric powered wheelchair driving using a position sensing joystick and an isometric joystick," Medical engineering \& physics, vol. 24, no. 10 pp. 703-708, 2002.

[13] H. P. Mahajan, B. E. Dicianno, R. A. Cooper, and D. Ding, "Assessment of wheelchair driving performance in a virtual reality-based simulator," The journal of spinal cord medicine, vol. 36, no. 4, pp. 322-332, 2013.

[14] I. A. Adelola, S. L. Cox, and A. Rahman, "Virtual environments for powered wheelchair learner drivers: Case studies," Technology and Disability, vol. 21, no. 3, pp. 97-106, 2009.

[15] N. Rodriguez, "Development of a wheelchair simulator for children with multiple disabilities," in 3rd IEEE VR International Workshop on Virtual and Augmented Assistive Technology (VAAT), March 2015, pp. 19-21.

[16] A. Alshaer, H. Regenbrecht, and D. OHare, "Immersion factors affecting perception and behaviour in a virtual reality power wheelchair simulator," Applied Ergonomics, vol. 58, pp. 1-12, 2017.

[17] U. Schultze, "Embodiment and presence in virtual worlds: a review," Journal of Information Technology, vol. 25, no. 4, pp. 434-449, 2010.

[18] M. Babel, F. Pasteau, S. Guégan, P. Gallien, B. Nicolas, B. Fraudet, S. Achille-Fauveau, and D. Guillard, "HandiViz project: clinical validation of a driving assistance for electrical wheelchair," in IEEE Workshop On Advanced Robotics And Its Social Impacts (ARSO), Lyon, France, Jul. 2015.

[19] L. Devigne, V. K. Narayanan, F. Pasteau, and M. Babel, "Low complex sensor-based shared control for power wheelchair navigation," in Intelligent Robots and Systems (IROS), 2016 IEEE/RSJ International Conference on. IEEE, 2016, pp. 5434-5439.

[20] T.-V. How, R. H. Wang, and A. Mihailidis, "Evaluation of an intelligent wheelchair system for older adults with cognitive impairments," Journal of neuroengineering and rehabilitation, vol. 10, no. 1, p. 90 , 2013.

[21] R. L. Kirby, D. J. Dupuis, A. H. MacPhee, A. L. Coolen, C. Smith, K. L. Best, A. M. Newton, A. D. Mountain, D. A. MacLeod, and J. P. Bonaparte, "The wheelchair skills test (version 2.4): measurement properties," Archives of physical medicine and rehabilitation, vol. 85 , no. 5, pp. 794-804, 2004.

[22] T. W. Schubert, "The sense of presence in virtual environments: A three-component scale measuring spatial presence, involvement, and realness." Zeitschrift für Medienpsychologie, vol. 15, no. 2, pp. 69-71, 2003.

[23] S. G. Hart, "Nasa-task load index (nasa-tlx); 20 years later," in Proceedings of the human factors and ergonomics society annual meeting, vol. 50, no. 9. Sage Publications Sage CA: Los Angeles, CA, 2006, pp. 904-908.

[24] S. Richir, S. Pineau, É. Monacelli, F. Goncalves, B. Malafosse, C. Dumas, A. Schmid, and J. Perret, "Design of portable and accessible platform in charge of wheelchair feedback immersion," in Virtual Reality (VR), 2015 IEEE. IEEE, 2015, pp. 389-390. 